\title{
Determinants in Tailoring Antidiabetic Therapies: A Personalized Approach
}

\author{
Aliya A. Rizvi ${ }^{1} \quad$ Mohammad Abbas $^{1} \quad$ Sushma Verma ${ }^{1}$ Shrikant Verma ${ }^{1}$ Almas Khan ${ }^{1}$ Syed T. Raza ${ }^{2}$ \\ Farzana Mahdi ${ }^{1}$ \\ 1 Department of Personalized and Molecular Medicine, Era University, \\ Lucknow, Uttar Pradesh, India \\ 2 Department of Biochemistry, Era University, Lucknow Medical \\ Address for correspondence Sushma Verma, PhD, Department of \\ Personalized and Molecular Medicine, Era University, Lucknow \\ 226003, Uttar Pradesh, India \\ College and Hospital, Lucknow, Uttar Pradesh, India \\ (e-mail: Sushma.verma919@gmail.com).
}

\begin{abstract}
Keywords

- diabetes

- precision medicine

- pharmacogenomics

- drug response

Diabetes has become a pandemic as the number of diabetic people continues to rise globally. Being a heterogeneous disease, it has different manifestations and associated complications in different individuals like diabetic nephropathy, neuropathy, retinopathy, and others. With the advent of science and technology, this era desperately requires increasing the pace of embracing precision medicine and tailoring of drug treatment based on the genetic composition of individuals. It has been previously established that response to antidiabetic drugs, like biguanides, sulfonylureas, dipeptidyl peptidase-4 (DPP-4) inhibitors, glucagon-like peptide 1 (GLP-1) agonists, and others, depending on variations in their transporter genes, metabolizing genes, genes involved in their action, etc. Responsiveness of these drugs also relies on epigenetic factors, including histone modifications, miRNAs, and DNA methylation, as well as environmental factors and the lifestyle of an individual. For precision medicine to make its way into clinical procedures and come into execution, all these factors must be reckoned with. This review provides an insight into several factors oscillating around the idea of precision medicine in type-2 diabetes mellitus.
\end{abstract}

\section{Introduction}

Diabetes refers to a hyperglycemic condition that may be caused either by a deficiency of insulin or defects in its action or both. Hyperglycemia that results from defects in insulin action, also referred to as insulin resistance, is called type-2 diabetes mellitus (T2DM) or noninsulin-dependent diabetes mellitus (NIDDM). ${ }^{1}$ According to the data provided by the International Diabetes Federation (IDF), there are approximately $74,194.7$ people aged between 20 and 79 years in India who had diabetes as of $2021 .^{2}$ Obesity is common in individuals suffering from T2DM and is believed to cause some degree of insulin resistance. ${ }^{3}$ A major cause of this metabolic disorder is a sedentary lifestyle and unhealthy

received

November 8, 2021

accepted

November 20, 2021
DOI https://doi.org/

10.1055/s-0041-1741109.

ISSN 2699-9404. eating habits. The early stages of this metabolic disorder can be controlled by proper diet and exercise, whereas later stages require treatment with antidiabetic drugs., 3

Physicians may prescribe these drugs alone (monotherapy) or in combination (combination therapy) according to the predisposition of the disease and in a set order. If unresponsiveness is observed with first-line therapy or toxicity occurs treatment shifts to subsequent lines of treatment according to the guidelines provided by the World Health Organization (WHO). ${ }^{5}$ Commercially available antidiabetic drugs include metformin, sulfonylureas (SU), glucagon-like peptide 1 (GLP-1) agonists, dipeptidyl peptidase-4 (DPP-4i) inhibitors, and others. There are several genes whose polymorphisms affect an individual's response to

\section{(c) 2022. The Author(s).}

This is an open access article published by Thieme under the terms of the Creative Commons Attribution License, permitting unrestricted use, distribution, and reproduction so long as the original work is properly cited. (https://creativecommons.org/licenses/by/4.0/)

Georg Thieme Verlag KG, Rüdigerstraße 14, 70469 Stuttgart, Germany 
these antidiabetic drugs including drug transporter genes like organic cation transporters (OCTs) viz., OCT1, OCT2, OCT3, multidrug and toxin extrusion transporter 1 (MATE1), drug-metabolizing genes like cytochrome P450 genes (CYP2C8, CYP2C9), and genes involved in drug action. The effect of genes on drug response is studied under "pharmacogenetics" which is established on the idea that an individual's genetic makeup and the structure, concentration and configuration of the proteins thus expressed, influences drug response in many ways. ${ }^{6,7}$ The structure and configuration of a protein depend on the genetic information and any variation in genes might alter the amino acid composition of the protein, folding of the protein, and structure of its active site. If the variation is present in the promoter region or its regulator genes, it will either upregulate its expression increasing the concentration or vice versa. Such variations in genes effectuate individuals to respond differently to certain drugs.

Epigenetic factors, like microRNAs, DNA methylation, and histone modifications, have also been studied to influence susceptibility to T2DM and response to antidiabetic drugs. Apart from genetic and epigenetic variations, there are other factors including environment, lifestyle, diet, and others, which determine susceptibility and drug response. These determinants concerning customization of antidiabetic therapies have been discussed in detail in this review.

\section{An Insight into Precision Medicine}

"Pharmacogenetics," a term coined by Vogel refers to the study of people's genetic variations which affect the way they respond to drugs. Differences in their genes affect the expression, structure, and configuration of the related proteins and this becomes the reason for different people responding differently to identical drug therapies. ${ }^{6,7}$ Several other factors, such as environmental factors, dietary habits, physical activity, age, sex, ethnicity, and other comorbidities, also cause a variable response to the same drug. ${ }^{8}$ Considering this variable response, it becomes necessary to introduce "precision medicine." Precision medicine refers to the use of medication, taking into account all the factors which can affect his response to therapeutics including his genetic makeup, lifestyle, and environmental factors because these, all together, are responsible for efficacy, neutrality, or toxicity of a particular drug. ${ }^{9}$ It has been shown that although metformin is used as first-line therapy for T2DM but some people respond positively whereas, in some, it causes adverse effects due to variations in related genes, and thus they require the use of alternate drugs. This is the reason that precision medicine is getting a lot of attention at present concerning disorders like T2DM, cancer, hypertension, and others. International HapMap project, which is an off-shoot of the Human Genome Project, aims to identify and catalog single nucleotide polymorphisms (or SNPs) which are sequences of genetic variants in diverse populations. ${ }^{10}$ The SNPs related to a particular disease can thus be identified, correlated with other factors (environment, lifestyle, etc.), and accordingly precision

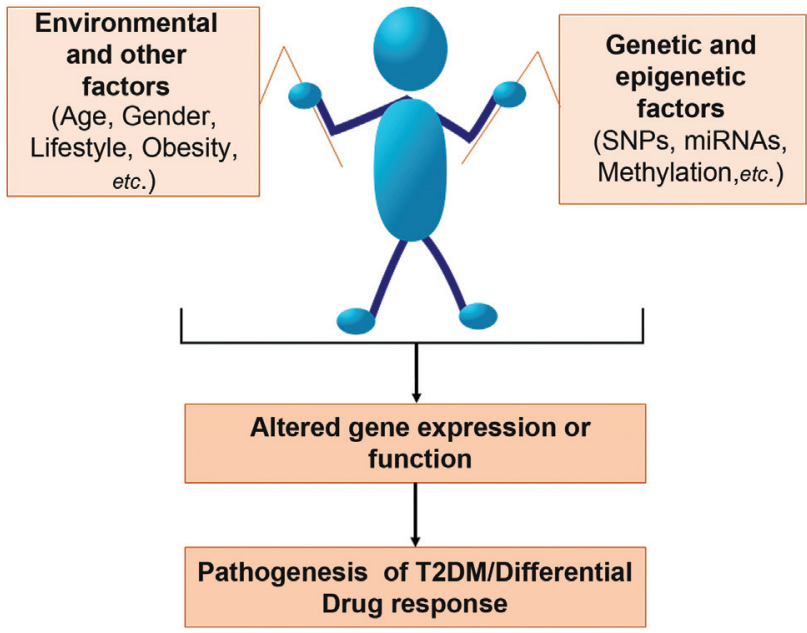

Fig. 1 Determinants of disease risk and pathogenesis as well as response to drug therapies. SNPs, single nucleotide polymorphisms; T2DM, type-2 diabetes mellitus.

medicine can be made available based on individual's genetic information (-Fig. 1).

\section{Genetic Markers for Type-2 Diabetes Mellitus}

Genetic variations may be protective against a particular disease or they can increase a person's susceptibility for a particular disease. Genetic markers for a disease are the genetic sequences or variations which may lead to its onset, progression, or severity. Some genetic markers also determine whether or not an individual will respond to a particular drug. By far, there are more than a hundred risk alleles that have been linked to T2DM in genome-wide association studies (GWAS). ${ }^{11}$ These alleles may influence $\beta$-cell function, insulin resistance, drug metabolization, drug transport, etc. Since T2DM is a heterogeneous and polygenic disease, identifying the most potent genetic biomarkers and applying the precision medicine approach is more difficult in the case of T2DM as compared with monogenic forms of diabetes. ${ }^{12}$

A study performed on 37,000 individuals identified 10 loci that reduced $\beta$-cell function and 3 that were linked to insulin sensitivity. The loci that were associated with reduced $\beta$-cell function were melatonin receptor 1B (MTNR1B), solute carrier family 30 member 8 (SLC30A8), thyroid adenoma-associated protein (THADA), transcription factor 7-like 2 TCF7L2, potassium voltage-gated channel subfamily $Q$ member 1 (KCNQ1), calcium/calmodulin-dependent protein kinase 1D (CAMK1D), etc. Three loci that were associated with insulin sensitivity were peroxisome proliferator-activated protein gamma (PPARG), fat mass and obesity-associated gene (FTO), and Kruppel-like factor 14 (KLF14). ${ }^{13}$ Metabolites, like glycine, lysophosphatidylcholine (LPC), and acetyl carnitine, have been previously known to have disturbed levels in patients with impaired glucose tolerance (IGT) ${ }^{14}$ and an in silico study showed an association between these metabolites and T2DM-associated genes, that is, insulin-like growth factor 1 (IGF1), insulin receptor substrate 1 (IRS1), insulin- 
degrading enzyme (IDE), TCF7L2, PPARG, and others. These genes were found to be efficient prediabetic markers in patients from United Arab Emirates (UAE). ${ }^{14}$ Among all the genetic markers, TCF7L2 is the largest susceptibility for T2DM and two of its variations, that is, rs7903746 and rs12255372, are the strongest and most efficient biomarkers. ${ }^{15-17}$ Some drug transporter genes like organic cation transporters (OCTS) have also been associated with increased T2DM susceptibility in several studies. ${ }^{18}$

\section{Genetic and Epigenetic Determinants of Response to Type-2 Diabetes Mellitus Therapeutics}

Metformin, an oral antidiabetic drug, is a biguanide and is used as the first-line therapy for T2DM. Second-line treatment involves use the of SU, whereas insulin or DPP-4 inhibitors, sodium-glucose cotransporter 2 (SGLT-2) inhibitors, or a thiazolidinediones form the third-line treatment as recommended by WHO. ${ }^{5}$ As previously discussed, the response to these drugs depends on alterations in the associated genes which include their transporters, metabolizers, and those involved in their action as well as their regulators. Genes, like OCT1, OCT2, OCT3, MATE1, MATE2, and others, are related to metformin response while cytochrome P450 (CYP) genes like CYP2C9, TCF7L2, potassium inwardly rectifying channel, subfamily J, member 11 (KCNJ11), etc., mainly influence SU response and response to DPP-4 inhibitors is altered by genes like $D P P-4, G L P-1$ receptor (GLP1R), KCNQ1, $K C N J 11$, cyclin-dependent kinase 5 regulatory-associated protein 1-like-1 (CDKAL1), and others. ${ }^{19}$ Similarly, response to a novel class of antidiabetic drugs, that is, GLP- 1 agonists is affected by TCF7L2 and the Wolframin endoplasmic reticulum transmembrane glycoprotein (WFS1), etc. ${ }^{20,21}$

\section{Genetic Determinants of Metformin Response}

Metformin is a derivative of guanidine, first discovered in the 1920 s in the Galega officinalis (French lilac) extracts. ${ }^{22}$ It does not require metabolizing enzymes for its action. ${ }^{23}$ The main site of action for metformin is the liver where it is taken up by its transporter genes like OCT1 and then by acting via adenosine monophosphate (AMP)-associated protein kinase (AMPK)-dependent pathway or AMPK-independent pathway, it alters ADP/AMP ratio thereby activating AMPK and inhibition of mitochondrial respiratory chain. ${ }^{23}$ The hepatic uptake and renal transport of metformin is primarily regulated by its transporter OCT1, and any alteration in this gene or protein thus expressed, directly affects metformin efficacy. It is coded by a highly polymorphic gene, SLC22A1, and these polymorphisms cause variable transport function. ${ }^{24,25}$ There are six common SNPs of OCT1 highly studied in association with T2DM. These are Met408Val (rs628031), Met420del (rs72552763), Phr160Leu (rs6383369), Pro341Leu (rs2282143), Gly401Ser (rs34130495), and an intronic SNP, rs622342. Concerning metformin response, Met408Val (rs628031) is the one massively studied SNP of OCT1 where AA genotype showed a significant reduction in HbA1c levels $(p<0.02)$ compared with those having heterozygous AG genotype in Han Chinese population after metformin therapy. ${ }^{26,27}$ The same variant has also been shown to counter the effect of Met420del variant where deletion of 3bp (GAT) reduces transport function of $O C T 1 .{ }^{24,28} \mathrm{High}$ association of intronic variant rs622342 with efficacy of metformin has been shown in which the major allele A had 5.6 fold higher chances to respond to metformin ${ }^{29}$ and the minor allele $C$ was associated with approximately $0.28 \%$ lower decrease in HbA1c levels. ${ }^{30}$ Slight reduction in function of OCT1 was observed with Pro341Leu and Phe160Leu. ${ }^{31,32}$

Five SNPs suggested for pharmacogenetic studies by the International Transporter Consortium are rs34130495 (Gly401Ser), rs12208357 (Arg61Cys), rs34059508 (Gly465Arg), rs55918055 (Cys88Arg), and rs72552763 (Met420del). Minor alleles of all of these SNPs, that is, A, T, A, C, respectively, and deletion in case of Met420del, reduce the expression of OCT1 gene and thus metformin effectiveness. ${ }^{33}$ Renal transport of metformin is mainly associated with OCT2, MATE1, and MATE2 genes. OCT2, which is majorly expressed in renal tubule cells, regulates entry of metformin into renal tubular cells. ${ }^{34}$ Minor allele of rs316019 of OCT2, that is, allele A, has been known to cause elevated plasma levels and reduced renal clearance of metformin due to decreased function of the transporter gene. ${ }^{35}$ Another study performed on African Americans, European Americans, Asians, and Mexicans showed better uptake of metformin with the same variant. ${ }^{36}$ Other variants of OCT2 like rs14540955, rs8177517, rs8177516, rs201919874, and rs8177507 had their variant alleles viz., T, G, T, T, and A, respectively, associated with reduced transporter activity. ${ }^{18}$ OCT3 is another major metformin transporter that is involved in its absorption and tissue distribution. ${ }^{37-39}$ Variant alleles of several exonic variants of OCT3 have been shown to reduce metformin uptake, viz., $\mathrm{T}$ alleles of rs8187725, rs1221246, and rs8187717 and G allele of rs8187722. ${ }^{18,40,41}$ Two other variants, that is, rs8187715 and rs2292334, studied separately showed better metformin efficacy with T allele of rs8187715 associated with increased uptake activity of the transporter and heterozygous variant (GA) of rs229334 showed high plasma concentration and lower elimination of the drug. ${ }^{40-43}$ MATE1 and MATE2 genes are associated with renal elimination of metformin. ${ }^{44}$ Minor allele, that is, A allele of rs2289669 and homozygous genotypes (CC and TT) of rs2252281 of MATE1 both showed better response with metformin monotherapy whereas homozygous genotypes (AA and GG) of rs 12943590 ( $-130 \mathrm{G}>\mathrm{A}$ ) of MATE2 conferred to reduced response by increasing renal clearance of metformin. ${ }^{45-48} \mathrm{G}$ allele of a polymorphism in plasma membrane monoamine transporter (PMAT) gene, i.e., rs3889348, caused gastrointestinal intolerance to metformin in several populations $^{49}$ (-Fig. 2).

\section{Genetic Determinants of Sulfonylureas Response}

If metformin alone fails to achieve desired reduction in HbA1c levels, it is recommended to combine it with other drugs like SU, DPP-4 inhibitors, or sodium-glucose cotransporter 2 (SGLT2) inhibitors as the first choice or TZDs, 


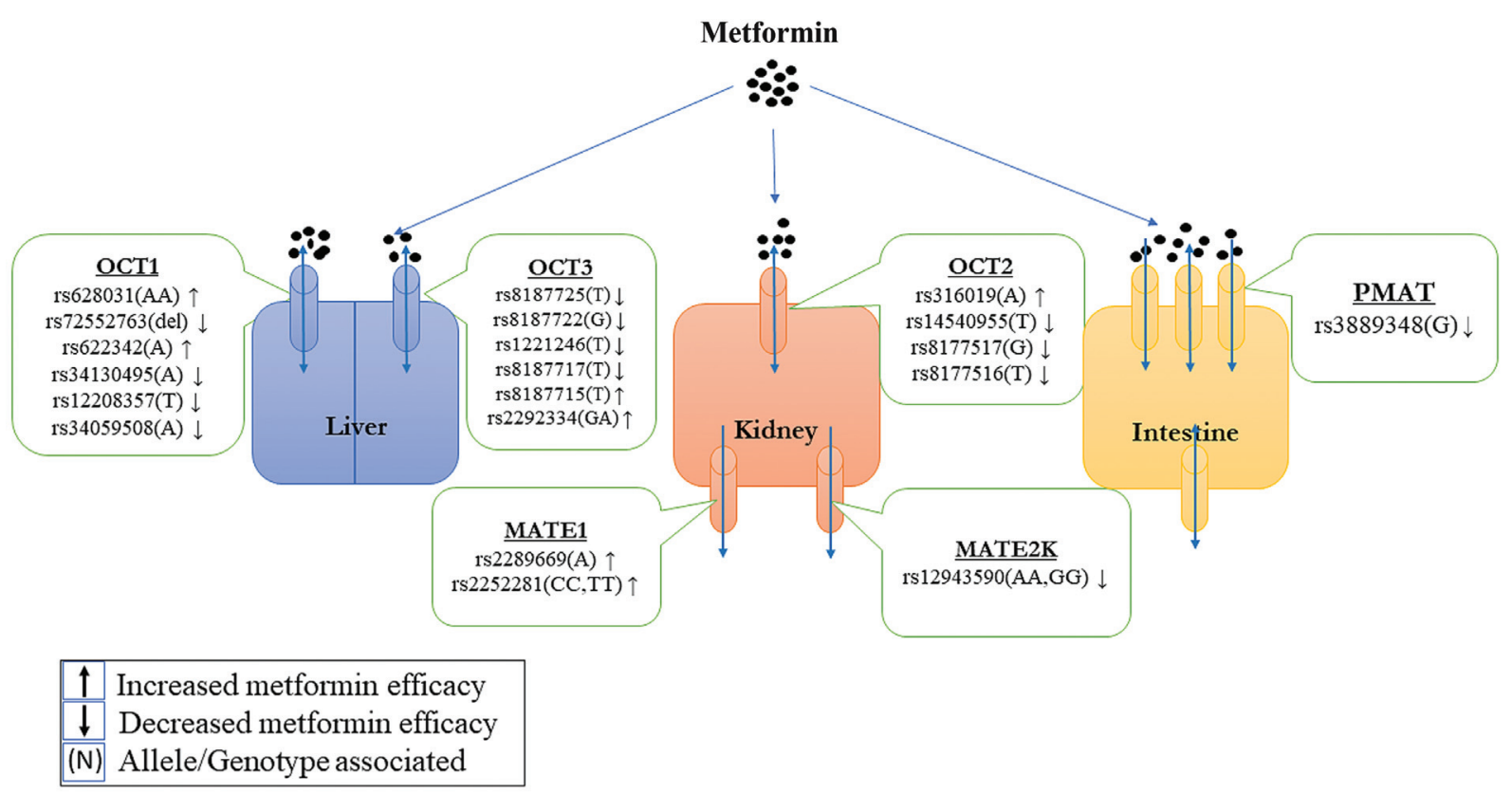

Fig. 2 Variations in transporter genes influencing metformin action in different organs. Given in brackets are the alleles/genotypes of the SNP that are associated with metformin response. (OCT1, 2, and 3, organic cation transporters 1, 2, and 3; MATE 1 and 2, multidrug and toxin extrusion transporters 1 and 2; PMAT, plasma membrane monoamine transporter.

glinides, or GLP1RA as second choice as directed by the Indian Council of Medical research and WHO.,50 SU works by activating potassium channels stimulating the release of insulin from $\beta$-cells of pancreas. ${ }^{51}$ Sulfonylurea receptor 1 (SUR1) presents on ATP-sensitive potassium channels (K-ATP) that is encoded by the ATP-binding cassette, subfamily $\mathrm{C}$, and member 8 ( $A B C C 8)$, whereas potassium inwardly rectifying channel, subfamily J, member 11 (KCNJ11) encodes pore subunit of $\mathrm{K}_{\text {ATP }}$ channel, Kir6.2. ${ }^{52}$ rs5219 of KCNJ11 is the most common SNP of this gene which is associated with improved response to SU in Chinese population; however, other studies have associated $\mathrm{T}$ allele of this variant with decreased expression of the subunit and increased risk of SU failure. ${ }^{53,54}$ Improved response to SU has been found in carriers of $G$ allele of nonsynonymous variant, rs757110 of ABCC8 gene. $^{54,55}$ SUs are dependent on drug metabolizing enzymes coded by genes, like cytochrome P450 genes, CYP2C9, CYP2C8, and others, for their metabolism. The main enzyme that metabolizes SU is CYP2C9. ${ }^{56}$ Variant alleles of two variations in this gene, that is, T allele of rs1799853 (CYP2C9*2) and C allele of rs1057910 (CYP2C9*3) are associated with poor response to SU since the protein expressed from these variants has reduced function. ${ }^{56-59}$ As for TCF7L2, apart from increasing the risk of T2DM, this gene also influences $S U$ response since it directly affects $\beta$-cell function. It was found that TT genotype of rs1225372 conferred to failure to attainment of lower level of HbA1c (i.e., 7\% or less) as compared with GG genotype $(p=0.006) .{ }^{60}$ Polymorphisms of two other genes which are T2DM susceptibility genes, that is, insulin receptor substrate-1 (IRS-1) and nitric oxide synthase 1 adaptor protein (NOS1AP), viz., rs1801278 (Gly972Arg) and rs10494366, respectively, also contribute to SU response. While AA and GA genotypes of rs1801278 of IRS-1 were associated with increased failure of
SU response, GT, and GG genotypes of rs10494366 of NOS1AP correlated with increased mortality rate in patients on $\mathrm{SU}^{61,62}$ (-Fig. 3).

\section{Genetic Determinants of Dipeptidyl Peptidase-4 Inhibitors}

DPP-4 enzyme degrades the hormones GLP-1 and glucosedependent insulinotropic polypeptide (GIP). ${ }^{63}$ DPP-4 inhibitors are the class of antidiabetic drugs that prevent the degradation of GLP-1 and GIP by DPP-4 thus decreasing the levels of glucagon and improving responsiveness of $\beta$-cells to increased glucose concentrations. ${ }^{64}$ These incretin hormones have a very short half-life and play a role in glucose-dependent insulin secretion, suppression of glucagon secretion, etc. ${ }^{65,66}$ There are several genes whose variations alter the responsiveness to DPP-4 inhibitors in individuals with T2DM, viz., DPP-4, GLP-1 receptor (GLP1R), KCNQ1, KCNJ11, CDKAL1, and others. ${ }^{67}$ DPP-4 is the substrate for DPP-4 inhibitors that bind to it so that inactivation of GLP-1 can be prevented. ${ }^{68}$ Efficacy of a DPP-4 inhibitor, sitagliptin, was found to be lower in individuals having TT genotype of rs2909451 and GG genotype of rs4664443 of the gene for DPP-4 enzyme. ${ }^{69}$ Studies have reported that homozygotes for A allele rs6923761 of GLP1R observed lower glucose-lowering effect of DPP-4 inhibitors, ${ }^{70,71}$ whereas A allele of rs3765467 was associated with better response to DPP-4 inhibitor therapy. ${ }^{71}$ Similarly, SNPs of KCNQ1 gene, which helps in GIP and GLP-1 secretion in intestines, $^{72}$ influence DPP-4 therapy whereas G allele of rs163184 conferred to lower response. ${ }^{73}$ CC genotype of a polymorphism rs2285676 in KCNJ11 gene, which stimulates insulin secretion from $\beta$-cells, ${ }^{74}$ showed two-fold higher probability of response to DPP-4 inhibitors. ${ }^{75}$ Two variants of CDKAL1, namely, rs7754840 (C > G) and rs756992 (A>G) had 


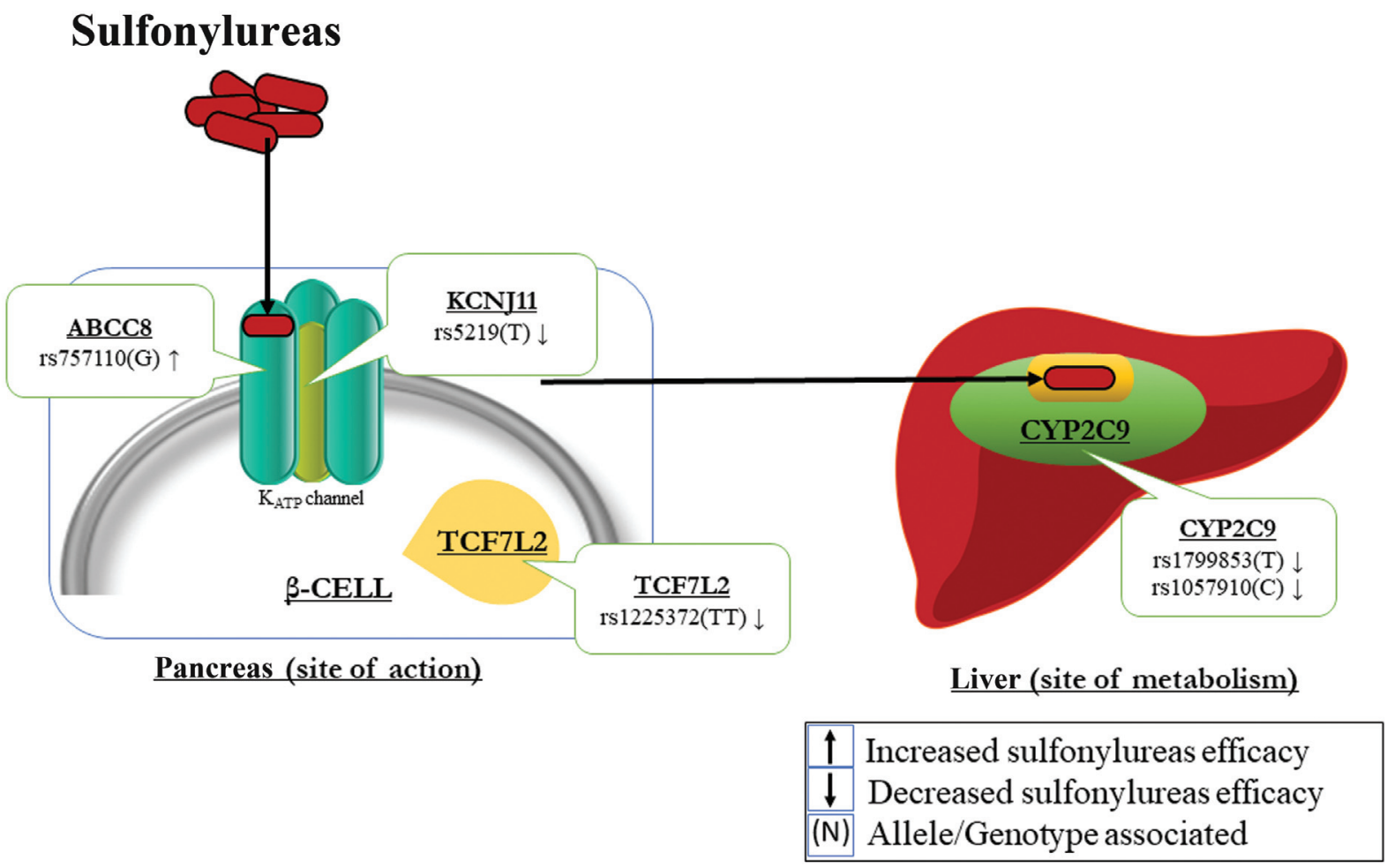

Fig. 3 Polymorphisms determining fate of sulfonylureas. Given in brackets are the alleles/genotypes of the SNPs that are associated with sulfonylureas response. ABCC8, ATP-binding cassette, subfamily C, member 8; CYP2C9, cytochrome P450; KCNJ11, potassium inwardly rectifying channel, subfamily J, member 11 ; TCF7L2, transcription factor 7-like2..

greater decrease in $\mathrm{HbA}_{1 \mathrm{c}}$ with at least one variant allele, that is, $\mathrm{G}$ and $\mathrm{A}$ allele, respectively, in comparison to those having common allele. ${ }^{76}$

\section{Genetic Determinants of Glucagon-Like Peptide-1 Agonists Response}

Recent guidelines issued by American Diabetes Association recommend GLP1 agonists as second line treatment for diabetic patients with cardiovascular disease or SGLT2 inhibitors if there are established comorbidities like chronic kidney disease, heart failure, and others, whereas SU, DPP4 inhibitors and TZDs are recommended as fourth line therapies after combination therapies and GLP-1 agonists. ${ }^{77}$ GLP1 agonists are a new class of antidiabetic medication. GLP1, as discussed earlier, is an incretin hormone that decrease the levels of glucagon, increases glucose-dependent insulin secretion. ${ }^{78}$ There are a few genes whose polymorphisms have studied to alter response to exogenous GLP-1 (GLP-1 agonists) like TCF7L2, WSF1, and GLP1R. Risk alleles of TCF7L2 and WFS1 variants, that is, rs7903146 (C > T) and rs10010131, respectively, were both significantly associated with reduced responsiveness to GLP-1 agonists, ${ }^{20,21}$ whereas variants of GLP1R, that is, rs3765467, rs761386, and rs587654, studied in Japanese population were not significantly associated with response to exogenous GLP-1. ${ }^{79}$

\section{Role of Epigenetics}

Epigenetic modifications are heritable changes that affect the expression of genes which include DNA methylation and histone modification (methylation, acetylation, SUMOylation, etc.). Most of these modifications correlate with repression of transcription like DNA and histone methylation, whereas some associate with upregulation of gene expression, for example, histone acetylation. ${ }^{80}$ MicroRNAs, or miRNAs, are another important epigenetic factor which regulate expression posttranscriptionally. Upon binding to the target mRNAs on their 3'-UTR (untranslated region), they destabilize mRNA, thereby repressing protein expression. ${ }^{81}$ Although all the epigenetic factors are important, there are fewer studies associating miRNAs and histone modifications with T2DM susceptibility, pathogenesis, and drug response as compared with DNA methylation which has been extensively studied. ${ }^{82}$

DNA methylation is the transfer of methyl group to cytosine ring catalyzed by DNA methyltransferases (DNMT) occurring mainly in those cytosine rich regions which are followed by a guanine. These regions are known as " $\mathrm{CpG}$ islands" which are usually unmethylated and more frequently found in the promoter region. DNA methylation is a method of controlling gene expression which mainly locks the genes in "off" position known as gene silencing. ${ }^{83-85}$ Lower expression of transporter genes of metformin, that is, OCT1, OCT3, and MATE1, as a result of DNA hypermethylation was observed in T2DM patients which reduced metformin uptake and transport. ${ }^{33,36,37}$ In addition to this, direct diminution of methylation of these transporter genes was observed with metformin. ${ }^{86}$ A study performed on Greek patients to investigate the relationship between promoter methylation of KCNJ11 and ABCC8 genes and hypoglycemia 
linked with SU concluded that methylation of only ABCC8 gene prevented the risk of SU-linked hypoglycemia. ${ }^{87}$ Yet another study associated hypermethylation of promoter of vault RNA 2-1 (VTRNA2-1) with better responsiveness to GLP-1 analogs. Also, A allele of a polymorphism in the same gene, that is, rs2346018, correlated with hypomethylation and thus reduced responsiveness to GLP-1 analogs. ${ }^{88}$

miRNAs have a role in posttranscriptional regulation of expression. These are 20 to 30 nucleotides-long noncoding RNAs which bind to 3'-UTR of the target primary transcript causing its destabilization and repression of translation. ${ }^{81,89}$ Several miRNAs have been associated with T2DM susceptibility, like miR-375, miR-146a, and others, which were overexpressed in T2DM patients. ${ }^{89}$ Apart from imparting susceptibility, some miRNAs have also been shown to be involved in action few antidiabetic drugs. One example of this is DPP-4 inhibitor linagliptin which alleviates kidney fibrosis by reversing the DDP-4-induced repression of miRNA29s. ${ }^{90}$ A study found that expression of KCNJ11 was influenced by rs60432575 G > A of MIR4532, hence causing reduction in sulfonylureas-mediated insulin secretion. ${ }^{91}$

\section{Other Factors Affecting Drug Response}

Apart from genetic and epigenetic variations, there are other factors that affect how an individual would respond to a particular drug. These factors include ethnicity, age, gender, lifestyle, obesity, and others. It has been widely accepted that obesity has a significant role in causing insulin resistance. Also, increasing age elevates the risk of T2DM susceptibility. Lifestyle factors including type and amount of stress, eating habits like intake of saturated fats, increasing weight, decreased amount of dietary fiber, decreased physical activity, smoking habits, alcohol intake, etc., alleviate a person's risk of developing T2DM and can also interfere with drug response. ${ }^{92}$ With respect to exercise, although it maintains blood-glucose level by increasing translocation of glucose transporter type 4 (GLUT4) and eventual uptake of glucose in active muscles, ${ }^{93}$ it has been observed that glucagon levels were the highest when exercise was combined with metformin therapy. ${ }^{94}$ In case of meglitinides and SU, there remains risk of hypoglycemia if combined with exercise and it is the highest with SU and insulin. When metformin was combined with exercise, hypoglycemia was found to occur only in individuals consuming excessive alcohol or those suffering from severe hepatic insufficiency. ${ }^{95,96}$ Therefore, it can be concluded that regular exercise might decrease T2DM risk but with respect to antidiabetic therapies, care must be taken, so that no adverse effects occur. Also, if some adjustments are made with dosage and exercise, the risk of hypoglycemia associated with some medications can be avoided. ${ }^{97}$

\section{Conclusion and Future Prospects}

T2DM, with a worldwide prevalence as high as $6.28 \%,{ }^{98}$ needs a hurried transition in its management from disease centric to explicitly patient centric. There are numerous studies already done and still going on concerning genetic factors for susceptibility to T2DM and personalizing diabetes treatment yet the pace of execution is excruciatingly slow. Tailoring drug treatment based on genetic profiles of can be procured by applying the knowledge obtained so far in modifying guidelines for diabetes care and management. As described in this review, environment, lifestyle (smoking habits, alcohol consumption, body weight, physical activity, etc.), genetic (SNPs, mutations, etc.), and epigenetic factors (DNA methylation, histone modifications, and miRNAs), and others influence drug response in several ways. These factors are variable in different individuals and the ultimate repercussion of these variations is on drug response or susceptibility and pathogenesis of the disease. Although there are limitations like T2DM being a highly heterogeneous disease, difficulty in implementing genetics-based care and treatment in some populations, etc., these can be subjugated by more studies performed on different populations and larger cohorts. With cost-effective genotyping assays, genetics-based health would be easier to embrace. Since it is more feasible to control T2DM in the early stages, there is also a dire need to screen all those at the risk of developing T2DM or going toward severity of the disease. Also, before the commencement of treatment, patients must be genotyped and the results must be correlated with lifestyle, clinical, environmental, and epigenetic aspects to differentiate them into responders and nonresponders, so that appropriate drugs and doses could be prescribed preventing any toxicity of antidiabetic drugs.

\section{Funding}

The work was supported by intramural funding of Era University, Lucknow, India.

\section{Conflict of Interest}

None declared.

\section{References}

1 American Diabetes Association. Diagnosis and classification of diabetes mellitus. Diabetes Care 2011;34(Suppl 1):S62-S69

2 International Diabetes Federation. IDF diabetes atlas, 9th ed. Accessed December 18, 2021 at: https://www.diabetesatlas.org/ data/en/country/93/in.html

3 Wolf AM, Conaway MR, Crowther JQ et al; Improving Control with Activity and Nutrition (ICAN) Study. Translating lifestyle intervention to practice in obese patients with type 2 diabetes: Improving Control with Activity and Nutrition (ICAN) study. Diabetes Care 2004;27(07):1570-1576

4 Nathan DM, Genuth S, Lachin J, et al; Diabetes Control and Complications Trial Research Group. The effect of intensive treatment of diabetes on the development and progression of long-term complications in insulin-dependent diabetes mellitus. N Engl J Med 1993;329(14):977-986

5 Guidelines on second-and third-line medicines and type of insulin for the control of blood glucose levels in non-pregnant adults with diabetes mellitus. Accessed December 6, 2021 at: https:// apps.who.int/iris/bitstream/handle/10665/272433/ 9789241550284-eng.pdf? sequence $=1$ \&isAllowed $=\mathrm{y}$

6 Motulsky AG. Drug reactions enzymes, and biochemical genetics. J Am Med Assoc 1957;165(07):835-837

7 Vogel F. Moderne probleme der humangenetik. In: Heilmeyer L, Schoen R, de Rudder B. Ergebnisse der inneren medizin und kinderheilkunde. Berlin, Germany: Springer; 1959:52-125 
8 American Diabetes Association. Standards of medical care in diabetes-2013. Diabetes Care 2013;36(Suppl 1):S11-S66

9 Cappelletti P. Medicina di precisione e medicina di laboratorio. La Rivista Italiana della Medicina di Laboratorio-Italian Journal of Laboratory Medicine 2016;12(03):129-133

10 International HapMap Consortium. The International HapMap Project. Nature 2003;426(6968):789-796

11 Xue A, Wu Y, Zhu Z, et al; eQTLGen Consortium. Genome-wide association analyses identify 143 risk variants and putative regulatory mechanisms for type 2 diabetes. Nat Commun 2018;9(01): 2941

12 Hattersley AT, Patel KA. Precision diabetes: learning from monogenic diabetes. Diabetologia 2017;60(05):769-777

13 Voight BF, Scott LJ, Steinthorsdottir V, et al; MAGIC investigators GIANT Consortium. Twelve type 2 diabetes susceptibility loci identified through large-scale association analysis. Nat Genet 2010;42(07):579-589

14 Wang-Sattler R, Yu Z, Herder C, et al. Novel biomarkers for pre-diabetes identified by metabolomics. Mol Syst Biol 2012; 8:615

15 Grant SF, Thorleifsson G, Reynisdottir I, et al. Variant of transcription factor 7-like 2 (TCF7L2) gene confers risk of type 2 diabetes. Nat Genet 2006;38(03):320-323

16 Tong Y, Lin Y, Zhang Y, et al. Association between TCF7L2 gene polymorphisms and susceptibility to type 2 diabetes mellitus: a large Human Genome Epidemiology (HuGE) review and metaanalysis. BMC Med Genet 2009;10:15

17 Pearson ER. Translating TCF7L2: from gene to function. Diabetologia 2009;52(07):1227-1230

18 Zazuli Z, Duin NJCB, Jansen K, Vijverberg SJH, Maitland-van der Zee AH, Masereeuw R. The impact of genetic polymorphisms in organic cation transporters on renal drug disposition. Int J Mol Sci 2020;21(18):6627

19 Becker ML, Pearson ER, Tkáč I Pharmacogenetics of oral antidiabetic drugs. Int J Endocrinol 2013;2013:686315

20 Schäfer SA, Tschritter O, Machicao F, et al. Impaired glucagon-like peptide-1-induced insulin secretion in carriers of transcription factor 7-like 2 (TCF7L2) gene polymorphisms . Diabetologia 2007; 50(12):2443-2450

21 Schäfer SA, Müssig K, Staiger $\mathrm{H}$, et al. A common genetic variant in WFS1 determines impaired glucagon-like peptide-1-induced insulin secretion. Diabetologia 2009;52(06):1075-1082

22 Pernicova I, Korbonits M. Metformin-mode of action and clinical implications for diabetes and cancer. Nat Rev Endocrinol 2014;10 (03):143-156

23 Todd JN, Florez JC. An update on the pharmacogenomics of metformin: progress, problems and potential. Pharmacogenomics 2014;15(04):529-539

24 Takane H, Shikata E, Otsubo K, Higuchi S, Ieiri I. Polymorphism in human organic cation transporters and metformin action. Pharmacogenomics 2008;9(04):415-422

25 Choi MK, Song IS. Organic cation transporters and their pharmacokinetic and pharmacodynamic consequences. Drug Metab Pharmacokinet 2008;23(04):243-253

26 Rena G, Hardie DG, Pearson ER. The mechanisms of action of metformin. Diabetologia 2017;60(09):1577-1585

27 Zhou Y, Ye W, Wang Y, et al. Genetic variants of OCT1 influence glycemic response to metformin in Han Chinese patients with type-2 diabetes mellitus in Shanghai. Int J Clin Exp Pathol 2015;8 (08):9533-9542

28 Giannoudis A, Wang L, Jorgensen AL, et al. The hOCT1 SNPs M420del and M408V alter imatinib uptake and M420del modifies clinical outcome in imatinib-treated chronic myeloid leukemia. Blood 2013;121(04):628-637

29 Umamaheswaran G, Praveen RG, Damodaran SE, Das AK, Adithan C. Influence of SLC22A1 rs622342 genetic polymorphism on metformin response in South Indian type 2 diabetes mellitus patients. Clin Exp Med 2015;15(04):511-517
30 Becker ML, Visser LE, van Schaik RH, Hofman A, Uitterlinden AG, Stricker BH. Interaction between polymorphisms in the OCT1 and MATE1 transporter and metformin response. Pharmacogenet Genomics 2010;20(01):38-44

31 Shu Y, Leabman MK, Feng B, et al; Pharmacogenetics Of Membrane Transporters Investigators. Evolutionary conservation predicts function of variants of the human organic cation transporter, OCT1. Proc Natl Acad Sci U S A 2003;100(10):5902-5907

32 Takeuchi A, Motohashi H, Okuda M, Inui K. Decreased function of genetic variants, Pro283Leu and Arg287Gly, in human organic cation transporter hOCT1. Drug Metab Pharmacokinet 2003;18 (06):409-412

33 Yee SW, Brackman DJ, Ennis EA, et al. Influence of transporter polymorphisms on drug disposition and response: a perspective from the international transporter consortium. Clin Pharmacol Ther 2018;104(05):803-817

34 Ito S, Kusuhara H, Yokochi M, et al. Competitive inhibition of the luminal efflux by multidrug and toxin extrusions, but not basolateral uptake by organic cation transporter 2, is the likely mechanism underlying the pharmacokinetic drug-drug interactions caused by cimetidine in the kidney. J Pharmacol Exp Ther 2012;340(02):393-403

35 Song IS, Shin HJ, Shim EJ, et al. Genetic variants of the organic cation transporter 2 influence the disposition of metformin. Clin Pharmacol Ther 2008;84(05):559-562

36 Kroetz DL, Yee SW, Giacomini KM. The pharmacogenomics of membrane transporters project: research at the interface of genomics and transporter pharmacology. Clin Pharmacol Ther 2010;87(01):109-116

37 Chen EC, Liang X, Yee SW, et al. Targeted disruption of organic cation transporter 3 attenuates the pharmacologic response to metformin. Mol Pharmacol 2015;88(01):75-83

38 Kekuda R, Prasad PD, Wu X, et al. Cloning and functional characterization of a potential-sensitive, polyspecific organic cation transporter (OCT3) most abundantly expressed in placenta. J Biol Chem 1998;273(26):15971-15979

39 Bleasby K, Castle JC, Roberts CJ, et al. Expression profiles of 50 xenobiotic transporter genes in humans and pre-clinical species: a resource for investigations into drug disposition. Xenobiotica 2006;36(10-11):963-988

40 Chen L, Pawlikowski B, Schlessinger A, et al. Role of organic cation transporter 3 (SLC22A3) and its missense variants in the pharmacologic action of metformin. Pharmacogenet Genomics 2010; 20(11):687-699

41 Sakata T, Anzai N, Kimura T, et al. Functional analysis of human organic cation transporter OCT3 (SLC22A3) polymorphisms. J Pharmacol Sci 2010;113(03):263-266

42 Lozano E, Briz O, Macias RIR, Serrano MA, Marin JJG, Herraez E. Genetic heterogeneity of SLC22 family of transporters in drug disposition. J Pers Med 2018;8(02):14

43 Hakooz N, Jarrar YB, Zihlif M, Imraish A, Hamed S, Arafat T. Effects of the genetic variants of organic cation transporters 1 and 3 on the pharmacokinetics of metformin in Jordanians. Drug Metab Pers Ther 2017;32(03):157-162

44 Motohashi H, Inui K. Organic cation transporter OCTs (SLC22) and MATEs (SLC47) in the human kidney. AAPS J 2013;15(02): 581-588

45 Becker ML, Visser LE, van Schaik RH, Hofman A, Uitterlinden AG, Stricker BH. Genetic variation in the multidrug and toxin extrusion 1 transporter protein influences the glucose-lowering effect of metformin in patients with diabetes: a preliminary study. Diabetes 2009;58(03):745-749

46 Mostafa-Hedeab G, Mohamed AA, Thabet G, et al. Effect of MATE 1, MATE 2 and OCT1 single nucleotide polymorphisms on metformin action in recently diagnosed egyptian type- 2 diabetic patients. Biomed Pharmacol J 2018;11(01):149-157

47 Stocker SL, Morrissey KM, Yee SW, et al. The effect of novel promoter variants in MATE1 and MATE2 on the pharmacokinetics 
and pharmacodynamics of metformin. Clin Pharmacol Ther 2013; 93(02):186-194

48 Choi JH, Yee SW, Ramirez AH, et al. A common 5'-UTR variant in MATE2-K is associated with poor response to metformin. Clin Pharmacol Ther 2011;90(05):674-684

49 Dawed AY, Zhou K, van Leeuwen N, et al; IMI DIRECT Consortium. Variation in the plasma membrane monoamine transporter (PMAT) (encoded by SLC29A4) and organic cation transporter 1 (OCT1) (encoded by SLC22A1) and gastrointestinal intolerance to metformin in type 2 diabetes: an IMI DIRECT study. Diabetes Care 2019;42(06):1027-1033

50 ICMR. ICMR guidelines for management of type 2 diabetes. . Accessed September 15, 2021: https://main.icmr.nic.in/sites/default/files/guidelines/ICMR_GuidelinesType2diabetes2018_0.pdf

51 Reis AF, Velho G. Sulfonylurea receptor -1 (SUR1): genetic and metabolic evidences for a role in the susceptibility to type 2 diabetes mellitus. Diabetes Metab 2002;28(01):14-19

52 Flanagan SE, Clauin S, Bellanné-Chantelot C, et al. Update of mutations in the genes encoding the pancreatic beta-cell $\mathrm{K}$ (ATP) channel subunits Kir6.2 (KCNJ11) and sulfonylurea receptor 1 (ABCC8) in diabetes mellitus and hyperinsulinism. Hum Mutat 2009;30(02):170-180

53 Sesti G, Laratta E, Cardellini M, et al. The E23K variant of KCNJ11 encoding the pancreatic beta-cell adenosine 5'-triphosphatesensitive potassium channel subunit Kir6.2 is associated with an increased risk of secondary failure to sulfonylurea in patients with type 2 diabetes. J Clin Endocrinol Metab 2006;91(06): 2334-2339

54 Fodor A, Cozma A, Suharoschi R, Sitar-Taut A, Roman G. Clinical and genetic predictors of diabetes drug's response. Drug Metab Rev 2019;51(04):408-427

55 Zhang H, Liu X, Kuang H, Yi R, Xing H. Association of sulfonylurea receptor 1 genotype with therapeutic response to gliclazide in type 2 diabetes. Diabetes Res Clin Pract 2007;77(01):58-61

56 Holstein A, Plaschke A, Ptak M, et al. Association between CYP2C9 slow metabolizer genotypes and severe hypoglycaemia on medication with sulphonylurea hypoglycaemic agents. Br J Clin Pharmacol 2005;60(01):103-106

57 Huang C, Florez JC. Pharmacogenetics in type 2 diabetes: potential implications for clinical practice. Genome Med 2011;3(11):76

58 Ragia G, Tavridou A, Elens L, Van Schaik RH, Manolopoulos VG. CYP2C9*2 allele increases risk for hypoglycemia in POR $* 1 /{ }^{*} 1$ type 2 diabetic patients treated with sulfonylureas. Exp Clin Endocrinol Diabetes 2014;122(01):60-63

59 Ragia G, Petridis I, Tavridou A, Christakidis D, Manolopoulos VG Presence of CYP2C9*3 allele increases risk for hypoglycemia in type 2 diabetic patients treated with sulfonylureas. Pharmacogenomics 2009;10(11):1781-1787

60 Jin T. The WNT signalling pathway and diabetes mellitus. Diabetologia 2008;51(10):1771-1780

61 Sesti G, Marini MA, Cardellini M, et al. The Arg972 variant in insulin receptor substrate- 1 is associated with an increased risk of secondary failure to sulfonylurea in patients with type 2 diabetes. Diabetes Care 2004;27(06):1394-1398

62 Becker ML, Aarnoudse AJ, Newton-Cheh C, et al. Common variation in the NOS1AP gene is associated with reduced glucoselowering effect and with increased mortality in users of sulfonylurea. Pharmacogenet Genomics 2008;18(07):591-597

63 Mentlein R, Gallwitz B, Schmidt WE. Dipeptidyl-peptidase IV hydrolyses gastric inhibitory polypeptide, glucagon-like peptide-1(7-36)amide, peptide histidine methionine and is responsible for their degradation in human serum. Eur J Biochem 1993; 214(03):829-835

64 Dalla Man C, Bock G, Giesler PD, et al. Dipeptidyl peptidase-4 inhibition by vildagliptin and the effect on insulin secretion and action in response to meal ingestion in type 2 diabetes. Diabetes Care 2009;32(01):14-18
65 Baggio LL, Drucker DJ. Biology of incretins: GLP-1 and GIP. Gastroenterology 2007;132(06):2131-2157

66 Kieffer TJ, McIntosh CH, Pederson RA. Degradation of glucosedependent insulinotropic polypeptide and truncated glucagonlike peptide 1 in vitro and in vivo by dipeptidyl peptidase IV. Endocrinology 1995;136(08):3585-3596

67 Rathmann W, Bongaerts B. Pharmacogenetics of novel glucoselowering drugs. Diabetologia 2021;64(06):1201-1212

68 Jamaluddin JL, Huri HZ, Vethakkan SR, Mustafa N. Pancreatic gene variants potentially associated with dipeptidyl peptidase- 4 inhibitor treatment response in type 2 diabetes. Pharmacogenomics 2014;15(02):235-249

69 Wilson JR, Shuey MM, Brown NJ, Devin JK. Hypertension and type 2 diabetes are associated with decreased inhibition of dipeptidyl peptidase-4 by sitagliptin. J Endocr Soc 2017;1(09):1168-1178

70 Ürgeová A, Javorský M, Klimčáková L, et al. Genetic variants associated with glycemic response to treatment with dipeptidylpeptidase 4 inhibitors. Pharmacogenomics 2020;21(05):317-323

71 Han E, Park HS, Kwon O, et al. A genetic variant in GLP1R is associated with response to DPP-4 inhibitors in patients with type 2 diabetes. Medicine (Baltimore) 2016;95(44):e5155

72 van Vliet-Ostaptchouk JV, van Haeften TW, Landman GW, et al. Common variants in the type 2 diabetes KCNQ1 gene are associated with impairments in insulin secretion during hyperglycaemic glucose clamp. PLoS One 2012;7(03):e32148

73 Gotthardová I, Javorský M, Klimčáková L, et al. KCNQ1 gene polymorphism is associated with glycaemic response to treatment with DPP-4 inhibitors. Diabetes Res Clin Pract 2017; 130:142-147

74 Haghvirdizadeh P, Mohamed Z, Abdullah NA, Haghvirdizadeh P, Haerian MS, Haerian BS. KCNJ11: genetic polymorphisms and risk of diabetes mellitus. J Diabetes Res 2015;2015:908152

75 Jamaluddin JL, Huri HZ, Vethakkan SR. Clinical and genetic predictors of dipeptidyl peptidase- 4 inhibitor treatment response in type 2 diabetes mellitus. Pharmacogenomics 2016;17(08): $867-881$

76 Osada UN, Sunagawa H, Terauchi Y, Ueda S. A common susceptibility gene for type 2 diabetes is associated with drug response to a DPP-4 inhibitor: pharmacogenomic cohort in Okinawa Japan. PLoS One 2016;11(05):e0154821

77 Doyle-Delgado K, Chamberlain JJ, Shubrook JH, Skolnik N, Trujillo J. Pharmacologic approaches to glycemic treatment of type 2 diabetes: synopsis of the 2020 American Diabetes Association's Standards of Medical Care in Diabetes Clinical Guideline. Ann Intern Med 2020;173(10):813-821

78 Drucker DJ, Nauck MA. The incretin system: glucagon-like peptide- 1 receptor agonists and dipeptidyl peptidase- 4 inhibitors in type 2 diabetes. Lancet 2006;368(9548):1696-1705

79 Lin $\mathrm{CH}$, Lee YS, Huang YY, Hsieh SH, Chen ZS, Tsai CN. Polymorphisms of GLP-1 receptor gene and response to GLP-1 analogue in patients with poorly controlled type 2 diabetes. J Diabetes Res 2015;2015:176949

80 McCarthy MI. Progress in defining the molecular basis of type 2 diabetes mellitus through susceptibility-gene identification. Hum Mol Genet 2004;13(Spec No 1):R33-R41

81 Cannell IG, Kong YW, Bushell M. How do microRNAs regulate gene expression? Biochem Soc Trans 2008;36(Pt 6):1224-1231

82 Jones PA. Functions of DNA methylation: islands, start sites, gene bodies and beyond. Nat Rev Genet 2012;13(07):484-492

83 Barres R, Zierath JR. DNA methylation in metabolic disorders. Am J Clin Nutr 2011;93(04):897S-900

84 Jin B, Li Y, Robertson KD. DNA methylation: superior or subordinate in the epigenetic hierarchy? Genes Cancer 2011;2(06): 607-617

85 Ban N, Yamada Y, Someya Y, et al. Hepatocyte nuclear factor1alpha recruits the transcriptional co-activator p300 on the GLUT2 gene promoter. Diabetes 2002;51(05):1409-1418 
86 García-Calzón S, Perfilyev A, Männistö V, et al. Diabetes medication associates with DNA methylation of metformin transporter genes in the human liver. Clin Epigenetics 2017;9:102

87 Karaglani M, Ragia G, Panagopoulou M, et al. Search for pharmacoepigenetic correlations in type 2 diabetes under sulfonylurea treatment. Exp Clin Endocrinol Diabetes 2019;127(04):226-233

88 Lin CH, Lee YS, Huang YY, Tsai CN. Methylation status of vault RNA 2-1 promoter is a predictor of glycemic response to glucagon-like peptide- 1 analog therapy in type 2 diabetes mellitus. BMJ Open Diabetes Res Care 2021;9(01):e001416

89 Zarkesh M, Ehsandar S, Hedayati M. Genetic and epigenetic aspects of type 2 diabetes mellitus: a review. Austin Endocrinol Diabetes Case Rep 2016;1(01):1004

90 Kanasaki K, Shi S, Kanasaki M, et al. Linagliptin-mediated DPP-4 inhibition ameliorates kidney fibrosis in streptozotocin-induced diabetic mice by inhibiting endothelial-to-mesenchymal transition in a therapeutic regimen. Diabetes 2014;63(06):2120-2131

91 Chen ZR, He FZ, Liu MZ, et al. MIR4532 gene variant rs60432575 influences the expression of KCNJ11 and the sulfonylureas-stimulated insulin secretion. Endocrine 2019;63(03):489-496

92 Tuomilehto J, Schwarz P, Lindström J. Long-term benefits from lifestyle interventions for type 2 diabetes prevention: time to expand the efforts. Diabetes Care 2011;34(Suppl 2): S210-S214

93 Colberg SR, Sigal RJ, Fernhall B, et al; American College of Sports Medicine American Diabetes Association. Exercise and type 2 diabetes: the American College of Sports Medicine and the American Diabetes Association: joint position statement. Diabetes Care 2010;33(12):e147-e167

94 Boulé NG, Robert C, Bell GJ, et al. Metformin and exercise in type 2 diabetes: examining treatment modality interactions. Diabetes Care 2011;34(07):1469-1474

95 Gulve EA. Exercise and glycemic control in diabetes: benefits, challenges, and adjustments to pharmacotherapy. Phys Ther 2008;88(11):1297-1321

96 Larsen JJ, Dela F, Madsbad S, Vibe-Petersen J, Galbo H. Interaction of sulfonylureas and exercise on glucose homeostasis in type 2 diabetic patients. Diabetes Care 1999;22(10):1647-1654

97 Shahar J, Hamdy O. Medication and exercise interactions: considering and managing hypoglycemia risk. Diabetes Spectr 2015;28 (01):64-67

98 Khan MAB, Hashim MJ, King JK, Govender RD, Mustafa H, Al Kaabi J. Epidemiology of type 2 diabetes - global burden of disease and forecasted trends. J Epidemiol Glob Health 2020;10(01):107-111 